\title{
FAITHFULLY EXACT FUNCTORS AND THEIR APPLICATIONS TO PROJECTIVE MODULES AND INJECTIVE MODULES
}

\author{
TAKESHI ISHIKAWA
}

\section{Introduction}

The aim of this paper is to study a property of a special kind of exact functors and give some applications to projective modules and injective modules.

In section 1 we introduce the notion of faithfully exact functors [Definition 1] as a generalization of the functor $T(X)=X \otimes M$, where $M$ is a faithfully flat module, and give a property of this class of functors [Theorem 1.1]. Next, applying this general theory to functors $\otimes$ and Hom, we define the notion of faithfully projective modules [Definition 2] and faithfully injective modules [Definition 3]. In the commutative case "faithfully projective" means, however, simply "projective and faithfully flat" [Proposition 2.3]. In section 2, equivalent conditions for a projective module $P$ to be faithfully projective are given [Theorem 2.2, Proposition 2.3 and 2.4]. And a simpler proof is given to Y. Hinohara's result [6] asserting that projective modules over an indecomposable weakly noetherian ring are faithfully flat [Proposition 2.5]. In section 3, we consider faithfully injective modules. Equivalent conditions for an injective module $Q$ to be faithfully injective are given [Theorem 3.1 and Cor.3.3]; e.g. $Q$ is faithfully injective if and only if $Q$ has a direct summand isomorphic to $E\left(\sum \oplus R / \mathfrak{m}_{\alpha}\right)$, where $\mathfrak{m}_{\alpha}$ ranges over all maximal ideals of $R$ and $E()$ denotes the injective envelope. In section 4 we study the annihilator relations between the ring $R$ and an $R$-injective module. Our faithfully injective modules are characterized in fact by the annihilator relation for ideals [Theorem 4.1]. Finally we give a generalization of E. Matlis' result [9], that is : when $R$ is a noetherian local ring with the maximal ideal $\mathrm{m}$ and $E(R / \mathrm{m})$ is not finitely generated, every proper submodule of $E(R / \mathrm{m})$ is finitely generated if and only if $R$ is analytically irreducible and Krull $\operatorname{dim} . R=1$ [Theorem 4.8].

Received April 13, 1963. 
Throughout this paper we assume that every ring has a unit element and every module is unitary. Further, modules and ideals mean left modules and left ideals, unless the contrary is stated.

\section{Faithfully exact functors}

Let $R$ be a ring. We consider a covariant or contravariant additive functor $T(X)$ defined on the category of (left or right) $R$-modules with values in some category of modules. First, we set the following

Definition 1. A functor $T$ is called a faithfully exact functor if the sequence $T(A) \stackrel{T(f)}{\longrightarrow} T(B) \stackrel{T(g)}{\longrightarrow} T(C)$ (or $T(C) \stackrel{T(g)}{\longrightarrow} T(B) \stackrel{T(f)}{\longrightarrow} T(A)$ in the contravariant case) is exact when and only when the sequence $A \stackrel{f}{\longrightarrow} B \stackrel{g}{\longrightarrow} C$ is exact.

Then, we have the following

THEOREM 1.1. Let $T(X)$ be an exact functor. Then the following conditions are equivalent:

(1) $T(X)$ is a faithfully exact functor.

(2) $T(A) \neq 0$ for every non zero $R$-module $A$.

(3) $T(\varphi) \neq 0$ for every non zero $R$-homomorphism $\varphi$.

(4) $T(R / a) \neq 0$ for every proper ideal a of $R$.

(5) $T(R / \mathfrak{m}) \neq 0$ for every maximal ideal $\mathfrak{m}$ of $R$.

Proof ${ }^{1)}$. We consider only the covariant case, since the contravariant case is quite similarly treated.

(1) $\Rightarrow(2):$ If $T(A)=0$, then, the sequence $T(0) \rightarrow T(A) \rightarrow T(0)$ is clearly exact. Hence, by (1), the sequence $0 \rightarrow A \rightarrow 0$ is exact. So $A=0$.

(2) $\Rightarrow(3)$ : Let $\varphi: X \rightarrow Y$ be an $R$-homomorphism. Then, since $T$ is an exact functor, we obtain the following commutative diagram with exact rows and columns:

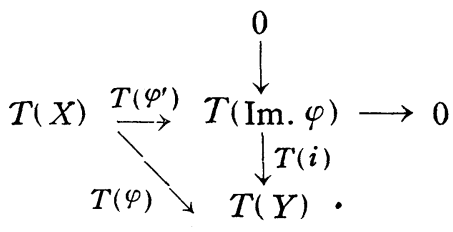

1) C.f. [3], Ch. 1, §3, Prop. 1 . 
where $i$ is the inclusion map and $\varphi$ is considered as the composition $X \stackrel{\varphi^{\prime}}{\longrightarrow}$ $\operatorname{Im} . \varphi \stackrel{i}{\longrightarrow} Y$. Now let $T(\varphi)=0$. Then, $T(i) T\left(\varphi^{\prime}\right)=T(\varphi)=0$ and so we have $T\left(\varphi^{\prime}\right)=0$. This implies $T(\operatorname{Im} . \varphi)=0$ and we have $\operatorname{Im} . \varphi=0$ by the condition (2). Thus $\varphi=0$.

(3) $\Longrightarrow(1):$ Let $T(A) \stackrel{T(f)}{\longrightarrow} T(B) \stackrel{T(g)}{\longrightarrow} T(C)$ be exact. It is to be proved that $A \stackrel{f}{\longrightarrow} B \stackrel{g}{\longrightarrow} C$ is exact. Since $T(g f)=T(g) T(f)=0$, we have $g f=0$ and $\operatorname{Im} . f$ $\subset$ Ker.g. Then we obtain the following commutative diagram with exact rows and columns:

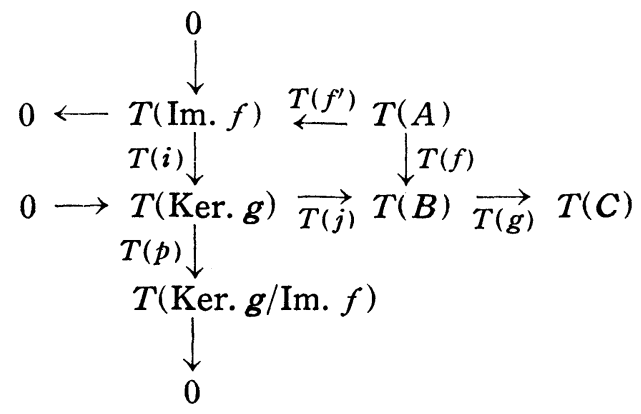

where $i$ and $j$ are inclusion maps and $p$ is the natural epimorphism. Since $T(g) T(j) x=0$ for any $x \in T(\operatorname{Ker} . g), T(j) x \in \operatorname{Ker} .(T(g))=\operatorname{Im} .(T(f))$. Thus there exists an element $y$ in $T(A)$ such that $T(f) y=T(j) x$. Hence $T(j) x=$ $T(f) y=T(j) T(i) T\left(f^{\prime}\right) y$ and so we have $x=T(i) T\left(f^{\prime}\right) y$. Therefore $T(i)$ is an epimorphism, hence $T(p)=0$ and by the assumption (3) we have $p=0$ which shows that Im. $f=$ Ker. $g$.

$(2) \Longrightarrow(4)$ and $(4) \Longrightarrow(5)$ are trivial.

$(5) \Longrightarrow(2)$ : Let $T(A)=0$. For each $a \in A, T(R a)=0$ since $T$ is exact. Now, if $l(a)=\{r \in R ; r a=0\} \neq R$, there exists a maximal ideal $\mathrm{m}$ containing $l(a)$ and we have an exact sequence $R / l(a) \rightarrow R / m \rightarrow 0$. Therefore $0=T(R a) \cong$ $T(R / l(a)) \rightarrow T(R / \mathrm{m}) \rightarrow 0$ is exact and this implies $T\left(R^{\prime} \mathrm{m}\right)=0$ which contradicts (5). Thus $l(a)=R$ i.e. $a=0$ and so $A=0$.

Now, we apply this theorem to the functors $\operatorname{Hom}_{R}$ and $\otimes_{R}$. In the subsequent sections we consider a fixed ring $R$ with identity 1 and sometimes speak of, dropping the symbol $R$, simply "module", "projective", "Hom", " $\otimes$ " e.t.c. instead of " $R$-module", " $R$-projective", "Hom ${ }_{R}$ ", " $\otimes_{R}$ " e.t.c., unless no confusion occurs. 


\section{Faithfully projective modules}

Let $T(X)=\operatorname{Hom}(P, X)$ and $U(X)=X \otimes M$, where $P$ and $M$ are fixed left $R$-modules. Then $T$ and $U$ are covariant additive functors defined on the category of left $R$-modules and right $R$-modules, respectively.

Definition 2. We say an $R$-module $P$ to be faithfully projective if $T$ is a faithfully exact functor. And an $R$-module $M$ is called faithfully flat if $U$ is a faithfully exact functor.

Applying Theorem 1.1 to the above functor $U$, we have the following well known theorem [3, Ch. 1, 3, Prop. 1].

TheOREM 2.1. Let $M$ be a flat left $R$-module. Then the following conditions are equivalent:

(1) $M$ is faithfully flat.

(2) $A \otimes M \neq 0$ for every non zero right $R$-module $A$.

(3) $\varphi \otimes 1_{M} \neq 0$ for every non zero right $R$-homomorphism $\varphi$, where $1_{M}$ denotes the identity map on $M$.

(4) $a M \neq M$ for every proper right ideal a of $R$.

(5) $\mathrm{m} M \neq M$ for every maximal right ideal $\mathfrak{m}$ of $R$.

Next, applying Theorem 1.1 to the above functor $T$, we have the following

Theorem 2.2. Let $P$ be a projective module. Then the following conditions are equivalent:

(1) $P$ is faithfully projective.

(2) $\operatorname{Hom}(P, A) \neq 0$ for every non zero left $R$-module $A$.

(3) $\operatorname{Hom}\left(1_{P}, \varphi\right) \neq 0$ for every non zero left $R$-homomorphism $\varphi$, where $1_{P}$ denotes the identity map on $P$.

(4) $\operatorname{Hom}(P, R / a) \neq 0$ for every proper left ideal a of $R$.

(5) $\operatorname{Hom}(P, R / m) \neq 0$ for every maximal left ideal $m$ of $R$.

Obviously a free module is faithfully projective and also faithfully flat. Now, we have the following

Proposition 2.3. If a module $P$ is faithfully projective, then $P$ is projective and faithfully flat. Further, when the ring $R$ is commutative or the module $P$ is finitely generated, the converse holds.

Proof. A module $P$, being faithfully projective, is projective and so flat. 
Now assume that $A \otimes_{R} P=0$ for a right $R$-module $A$. Then it is to be proved $A=0$ (Theorem 2.1 (2)). By [4, II. 5. 2] for any $Z$-module $C$ we have $\mathrm{Hom}_{R}$ $\left(P, \operatorname{Hom}_{Z}(A, C)\right) \cong \operatorname{Hom}_{Z}\left(A \otimes_{R} P, C\right)=0$, where $Z$ denotes the ring of integers. Since $P$ is faithfully projective, we have $\operatorname{Hom}_{Z}(A, C)=0$ for any $Z$-module $C$ by Theorem $2.2(2)$, and hence $A=0$.

In the finitely generated case, we can prove the converse by utilizing a similar formula as above given in [4, VI. 5. 2].

Finally let $R$ be commutative and $P$ be projective and faithfully flat. Then $P / \mathfrak{m} P \neq 0$ for any maximal ideal $\mathfrak{m}$ of $R$ by Theorem 2.1 (5). Therefore $\operatorname{Hom}_{R}(P / \mathfrak{m} P, R / \mathrm{m})=\operatorname{Hom}_{R / \mathfrak{m}}(P / \mathfrak{m} P, R / \mathrm{m}) \neq 0$ and we have an exact sequence $0 \rightarrow \operatorname{Hom}(P / \mathrm{m} P, R / \mathrm{m}) \rightarrow \operatorname{Hom}(P, R / \mathfrak{m})$. This implies $\operatorname{Hom}(P, R / \mathfrak{m}) \neq 0$ and $P$ is faithfully projective by Theorem 2.2 (5).

Next, we give a further characterization of faithfully projective modules.

Proposition 2.4. Let $P$ be a projective module. Then the following conditions are equivalent:

(1) $P$ is faithfully projective.

(2) There exists a positive integer $n$ such that $P_{1} \oplus \cdots \oplus P_{n}\left(\right.$ where $\left.P_{i}=P\right)$

has a direct summand isomorphic to the ring $R$.

(3) There exists an index set I of infinite cardinal such that $\sum_{i \in I} \oplus P_{i}$ (where $P_{i}=$ P) is free.

Further, when $R$ is semi-local, we can take $n=1$ in the case (2).

Proof. (1) $\Longrightarrow(2)$ : Let $P$ be a faithfully projective module. Then, by Theorem $2.2(5)$, for every maximal left ideal $\mathrm{m}_{\alpha}$ of $R$, there exists a homomorphism $f_{\alpha}: P \rightarrow R$ such that $f_{\alpha}(P) \nsubseteq \mathrm{m}_{\alpha}$. Therefore we have $1=\sum_{i=1}^{n} f_{\alpha_{i}}\left(p_{i}\right)$ for some $p_{i} \in P$, since $R=\bigcup_{\alpha} f_{\alpha}(P)$. Now we define a homomorphism $f: P_{1} \oplus \cdots \oplus P_{n}$ $\rightarrow R$ by $f\left(p_{1}^{\prime}, \ldots, p_{n}^{\prime}\right)=\sum_{i=1}^{n} f_{\alpha_{i}}\left(p_{i}^{\prime}\right)$, then $f$ is obviously an epimorphism. Hence $P_{1} \oplus \cdots \oplus P_{n}$ has a direct summand isomorphic to $R$.

When $R$ is semi-local, we can easily find an element $p$ of $P$ such that $f_{i}(p) \notin m_{i}$ for each $i$. Therefore $f=\sum f_{i}: P \rightarrow R$ is an epmorphism.

$(2) \Rightarrow(3)$ : Take $I$ to be an infinite index set of which power is equal to that of generators of $P$. (Take $I$ to be countable if $P$ is finitely generated). Then $\sum_{i \in I} \oplus P_{i}$ has, by (2), a free direct summand with a basis of same power as $I$. Thus this is free by Eilenberg's lemma [c.f. 7]. 
(3) $\Rightarrow(1)$ : Let $\sum_{i \in I} \oplus P_{i}$ be free as in (3). Then $\prod_{i \in I} \operatorname{Hom}\left(P_{i}, R / \mathrm{m}\right) \cong$ Hom $\left(\sum_{i \in I} \oplus P_{i}, R / \mathrm{m}\right) \neq 0$. Hence $\operatorname{Hom}(P, R / \mathrm{m}) \neq 0$ and thus $P$ is faithfully projective by Theorem 2.2 (5).

It is known that any finitely generated projective module over an indecomposable commutative ring is faithfully flat [5]. Recently Y. Hinohara [6] proved that if $R$ is an indecomposable commutative ring and the maximal spectrum of $R$ is a noetherian space, then any projective module is faithfully flat. Now we give a somewhat simple (but essentially same) proof of these facts and moreover we prove, dropping the noetherian assumption, that any projective module over an indecomposable commutative ring is almost faithfully flat in a sense. That is

Proposition 2.5. Let $R$ be an indecomposable commutative ring and $P$ be a non zero projective module over $R$. Then we have

(1) If $P$ is finitely generated, $P$ is faithfully projective. (O. Goldman)

(2) If the maximal spectrum of $R$ is a noetherian space, $P$ is faithfully projective. (Y. Hinohara)

(3) When $R$ is not semi-local (the semi-local case is included in (2)), there exist infinitely many maximal ideals $\mathrm{m}_{\alpha}$ such that $P \neq \mathrm{m}_{\alpha} P$.

Proof. We use the following notations:

$X$ : the maximal spectrum of $R$. (for definition c.f. [3])

$V(a)=\{m \in X ; a \subset m\}$, where $a$ is an ideal of $R$.

$S(P)=\{\mathrm{m} \in X ; \operatorname{Hom}(P, R / \mathrm{m})=0$ or equivalently $P=\mathrm{m} P\}$.

$T(P)=\{\mathrm{m} \in X ; \operatorname{Hom}(P, R / \mathrm{m}) \neq 0$ or equivalently $P \neq \mathrm{m} P\}=X-\mathrm{S}(P)$.

We use the following without proof :

Lemma 2.6. (Y. Hinohara [6]) $X$ is a noetherian space if and only if for any ideal a there exists a finitely generated ideal $\mathfrak{b}$ which is contained in a such that $V(\mathfrak{a})=V(\mathfrak{b})$.

We return to prove the proposition. Let $P$ be not faithfully projective i.e. $S(P) \neq \phi$. If $T(P)=\phi$, then $P=m P$ for every $m \in X$. This implies $P=0$ by $[6$, Lemma 1.4] and $[10, \S 9$, Lemma 2], since $P$ is projective. Therefore we may assume that also $T(P) \neq \phi$. For each $\mathfrak{n}_{\alpha} \in T(P)$, there exists a homomorphism $f_{\alpha}: P \rightarrow R$ such that $f_{\alpha}(P) \nsubseteq \mathfrak{n}_{\alpha}$, since $\operatorname{Hom}\left(P, R / \mathfrak{n}_{\alpha}\right) \neq 0$. Therefore there exist 
elements $p_{\alpha}$ of $P$ such that $f_{\alpha}\left(p_{\alpha}\right) \notin \mathfrak{n}_{\alpha}$. Let $a$ be the ideal generated by the elements $f_{\alpha}\left(p_{\alpha}\right)$ 's. Then $a \nsubseteq \mathfrak{n}_{\alpha}$ for any $\mathfrak{n}_{\alpha} \in T(P)$. On the other hand if $\mathfrak{m}_{\alpha}$ is an element of $S(P)$, then $f(P) \subset \mathrm{m}_{\alpha}$ for any homomorphism $f: P \rightarrow R$. Hence we have $a \subset \mathfrak{m}_{\alpha}$ for any $\mathfrak{m}_{\alpha} \in S(P)$. Thus $V(\mathfrak{a})=S(P)$ and $X-V(\mathfrak{a})=T(P)$. If $m \in S(P), P_{\mathfrak{m}}=0$ and hence, for any $p \in P$ there exists an element $s$ of $R-m$ such that $s p=0$. Now assume that $P$ is moreover finitely generated or $T(P)$ is a finite set. Then we can find easily an element $s_{\alpha} \in R-\mathfrak{m}_{\alpha}$ for each $\mathfrak{m}_{\alpha} \in$ $S(P)$ such that $s_{\alpha} \mathfrak{a}=0$. Let $\mathfrak{b}$ be the ideal generated by these $s_{\alpha}$ 's. Then $\mathfrak{b a}=0$ and $\mathfrak{b}+\mathfrak{a}=R$, since $\mathfrak{b} \leftarrow \mathfrak{m}_{\alpha}$ for any $m_{\alpha} \in S(P)$ and $a \nsubseteq \mathfrak{n}_{\alpha}$ for any $\mathfrak{n}_{\alpha} \in T(P)$. Thus we have $R=\mathfrak{b} \oplus \mathfrak{a}$, which contradicts the indecomposability of $R$. Thus (1) and (3) are proved. As to the case of (2), we can have a finitely generated ideal $a^{\prime} \subset a$ such that $V\left(a^{\prime}\right)=V(a)$ by Lemma 2.6. Therefore we can proceed quite similarly to find an ideal $\mathfrak{b}$ such that $\mathfrak{b} \oplus \mathfrak{a}^{\prime}=R$.

\section{Faithfully injective modules}

In place of $T(X)=\operatorname{Hom}(P, X)$ in section 2, let us now consider the contravariant functor $V(X)=\operatorname{Hom}(X, Q)$, where $Q$ is a fixed $R$-module. First, we will set the following

Definition 3. An $R$-module $Q$ is called faithfully injective if $V$ is a faithfully exact functor.

Then, applying Theorem 1.1 to this functor, we have immediately the following

TheоRем 3.1. Let $Q$ be an injective module. Then the following conditions are equivalent:

(1) $Q$ is faithfully injective.

(2) $\operatorname{Hom}(A, Q) \neq 0$ for every non zero left $R$-module $A$.

(3) $\operatorname{Hom}\left(\varphi, 1_{Q}\right) \neq 0$ for every non zero left $R$-homomorphism $\varphi$, where $1_{Q}$ denotes the identity map on $Q$.

(4) $\operatorname{Hom}(R / \mathfrak{a}, Q) \neq 0$ for every proper left ideal a of $R$.

(5) $\operatorname{Hom}(R / \mathrm{m}, Q) \neq 0$ for every maximal left ideal in of $R$.

From this, we can easily show the following Corollary 3.2 and 3.3.

Corollary 3.2 .

(1) If $Q$ is faithfully injective and $Q^{\prime}$ is injective, then $Q \oplus Q^{\prime}$ is faithfully in- 
jective.

(2) Let $E=E\left(\sum_{\alpha} \oplus R / \mathrm{m}_{\alpha}\right)$, where $\mathrm{m}_{\alpha}$ ranges over all maximal left ideals of $R$ and $E()$ denotes the injective envelope ${ }^{1)}$. Then $E$ is a faithfully injective module.

This injective module $E$ is called the canonical injective (left) module. From now on, $E$ always denotes this injective module. Note that if $R$ is semilocal or noetherian, then $E \cong \sum_{\alpha} \oplus E\left(R / \mathrm{m}_{\alpha}\right)$ by [8, Prop. 2.1].

Corollary 3.3. Let $Q$ be an injective module and $E$ be the canonical injective module. Then the following conditions are equivalent:

(1) $Q$ is faithfully injective.

(2) $Q$ contains an isomorphic image of every simple $R$-module.

(3) $Q$ has a direct summand isomorphic to $E$.

Remark. Azumaya [1] calls a module $Q$ (not necessary injective) distinguished if it has the property (2) of Corollary 3.3. If $R$ is a commutative semi-local ring and $\mathfrak{n}$ is its Jacobson radical, then obviously $E \cong E(R / \mathfrak{n})$. Azumaya [1] calls $E(R / \mathfrak{n})$ the canonical $R$-module when $R$ is a commutative ring with minimum condition.

Proposition 3.4. Let $R$ be a commutative ring. Suppose that every non zero injective $R$-module is faithfully injective. Then $R$ is a primary ring (i.e. $R$ has only one proper prime ideal). Moreover the converse holds when $R$ is noetherian.

Proof. Let $\mathfrak{p}$ be a prime ideal of $R$. Then $E(R / \mathfrak{p})$ is faithfully injective and therefore we have $\operatorname{Hom}(R / \mathrm{m}, E(R / \mathfrak{p})) \neq 0$ for any maximal ideal $\mathrm{m}$ of $R$. Let $f$ be a non zero element of $\operatorname{Hom}(R / \mathrm{m}, E(R / \mathfrak{p}))$. Then $R / \mathrm{m} \cong f(R / \mathrm{m})$ is a non zero submodule of $E(R / \mathfrak{p})$, so $f(R / \mathfrak{m}) \cap R / \mathfrak{p} \neq 0$ since $E(R / \mathfrak{p})$ is an essential extension of $R / \mathfrak{p}$. Therefore, for a non zero element $x$ of $f(R / \mathrm{m}) \cap R / \mathfrak{p}$, we have $\mathfrak{m}=0(x)=\mathfrak{p}$, where $0(x)$ denotes the oder ideal of $x$. Thus $R$ has only one proper prime ideal.

Conversely, if $R$ is noetherian, every injective module is then a direct sum of indecomposable injective modules [8, Th. 2.5] and every indecomposable injective module is isomorphic to $E(R / \mathfrak{p})$ for some prime ideal $p$ [8, Th. 3.1].

1) For the definition and fundamental properties of the injective envelope, see [8]. 
Therefore, if $R$ has only one proper prime ideal $\mathrm{m}$, every injective module has a direct summand isomorphic to $E=E(R / \mathrm{m})$ and hence faithfully injective by Corollary 3.3.

Proposition 3.5. Let $R$ be a cmmutative noetherian ring. Then an $R$ module $Q$ is faithfully injective if and only if $Q_{\mathfrak{m}}$ is faithfully injective as an $R_{\mathfrak{m}}$-module for every maximal ideal $\mathfrak{m}$ of $R$.

Proof. By [2, Cor. 1.4], $\boldsymbol{Q}$ is injective if and only if $\boldsymbol{Q}_{\mathfrak{m}}$ is $R_{\mathfrak{m}}$-injective for every maximal ideal $\mathrm{m}$. If $\mathrm{m}, \mathrm{m}^{\prime}$ are two distinct maximal ideals of $R$, then we have $\operatorname{Hom}_{R}(R / \mathfrak{m}, Q)_{\mathfrak{m}} \cong \operatorname{Hom}_{R_{\mathfrak{m}}}\left(R_{\mathfrak{m}} / \mathfrak{m} R_{\mathfrak{m}}, Q_{\mathfrak{m}}\right)=0$. Therefore, for a fixed maximal ideal $\mathfrak{m}$ we have: $\operatorname{Hom}(R / \mathfrak{m}, Q)=0 \Leftrightarrow \operatorname{Hom}(R / \mathfrak{m}, Q)_{\mathfrak{m}_{\alpha}}=0$ for every maximal ideal $\mathrm{m}_{\alpha} \Leftrightarrow \operatorname{Hom}(R / \mathfrak{m}, Q)_{\mathfrak{m}}=0 \Leftrightarrow \operatorname{Hom}_{R_{\mathfrak{m}}}\left(R_{\mathfrak{m}} / \mathrm{m} R_{\mathfrak{m}}, Q_{\mathfrak{m}}\right)=0$. Hence $\operatorname{Hom}(R / \mathrm{m}, Q) \neq 0$ for every maximal ideal $m$ if and only if $\operatorname{Hom}_{R_{\mathfrak{m}}}$ $\left(R_{\mathfrak{m}} / \mathfrak{m} R_{\mathfrak{m}}, Q_{\mathfrak{m}}\right) \neq 0$ for every maximal ideal $\mathrm{m}$. This concludes the proof.

Finally we will state a proposition without detailed proof.

Proposition 3.6. Let $R$ be a commutative ring and $Q$ be a faithfully injective module. Then, a module $M$ is flat (resp. faithfully flat) if and only if $\operatorname{Hom}(M, Q)$ is injective (resp. faithfully injective).

Proof. This is an immediate consequence of natural equivalences of [4, II 5.2. and VI 5.1] and Theorem 3.1.

\section{Annihilator relations}

Throughout this section we assume that $R$ is a commutative ring and $E$ is the canonical injective module. Let $Q$ be an injective module, $N$ be its submodule and $a$ be an ideal of $R$. We will denote the annihilator of $a$ in $Q$ by $\mathfrak{a}^{*}$ and the annihilator of $N$ in $R$ by $N^{*}$. That is :

$$
\begin{aligned}
& a^{*}=\operatorname{Ann}_{Q}(\mathfrak{a})=\{x \in Q ; \mathfrak{a} x=0\} \\
& N^{*}=\operatorname{Ann}_{R}(N)=\{r \in R ; r N=0\}
\end{aligned}
$$

Generally we have $a \subset \mathfrak{a}^{* *}$ and $N \subset N^{* *}$ for any ideal $a$ of $R$ and any submodule $N$ of $Q$. If $a=a^{* *}$ (resp. $N=N^{* *}$ ) holds, we say that the annihilator relation holds for a (resp. for $N$ ). If the annihilator relations hold for any ideal of $R$ and for any submodule of $Q$, we say that the annihilator relations hold between $R$ and $Q$. It is known that if $R$ has the minimum condition for ideals [1] or $R$ is a complete noetherian (semi-) local ring [8], the annihilator 
relations hold between $R$ and $E$.

Now we will show that the annihilator relation for ideals characterizes the faithfully injectivity of an injective module.

Theorem 4.1. Let $Q$ be an injective module. Then the following conditions are equivalent:

(1) $Q$ is faithfully injective.

(2) $\mathfrak{a}=\mathfrak{a}^{* *}$ for every ideal $\mathfrak{a}$ of $R$.

(3) $m=m^{* *}$ for every maximal ideal $m$ of $R$.

Proof. (1) $\Longrightarrow(2)$ : In general, we have easily $\mathfrak{a} \subset \mathfrak{a}^{* *}$ and $\mathfrak{a}^{*}=\mathfrak{a}^{* * *}$. From the exact sequence $0 \rightarrow a^{* *} / \mathfrak{a} \rightarrow R / \mathfrak{a} \rightarrow R / \mathfrak{a}^{* *} \rightarrow 0$, we obtain the following exact sequence:

$$
0 \rightarrow \operatorname{Hom}\left(R / \mathfrak{a}^{* *}, Q\right) \rightarrow \operatorname{Hom}(R / \mathfrak{a}, Q) \rightarrow \operatorname{Hom}\left(\mathfrak{a}^{* *} / \mathfrak{a}, Q\right) \rightarrow 0
$$

By the well known isomorphism, we have $\operatorname{Hom}(R / \mathfrak{a}, Q) \cong \mathfrak{a}^{*}=\mathfrak{a}^{* * *} \cong \operatorname{Hom}$ $\left(R / a^{* *}, Q\right)$ and hence $\operatorname{Hom}\left(a^{* *} / \mathfrak{a}, Q\right)=0$. Since $Q$ is faithfully injective, we obtain $\mathfrak{a}^{* *} / \mathfrak{a}=0$ i.e. $a=a^{* *}$.

(2) $\Rightarrow(3)$ : trivial.

$(3) \Longrightarrow(1)$ : If $\operatorname{Hom}(R / \mathrm{m}, Q) \cong \mathrm{m}^{*}=0$, then $\mathrm{m}=\mathrm{m}^{* *}=0^{*}=R$. Thus Hom $(R / m, Q) \neq 0$ for every maximal ideal $m$ of $R$ and this implies that $Q$ is faithfully injective.

Proposition 4.2. Let $Q$ be an injective module. If the annihilator relations hold between $R$ and $Q$, then $Q$ is isomorphic to $E$.

Proof $^{1)}$. By Theorem 4.1, $Q$ is faithfully injective. Then by Cor. 3.3, $Q$ has a direct summand $E^{\prime}$ isomorphic to $E$. Thus we have $Q=E^{\prime} \oplus Q^{\prime}$ for some injective submodule $Q^{\prime}$. It is to be proved $Q^{\prime}=0$. Let $Q^{\prime} \neq 0$ or more generally let $N$ be any non zero submodule of $Q$. By the assumption we have $N=N^{* *}$. But there exists a maximal ideal $m$ such that $N^{*} \subset m$ and hence we get $N=$ $N^{* *} \supset \mathrm{m}^{*}$. Therefore $N \subset E^{\prime} \neq 0$ and especially $Q^{\prime} \cap E^{\prime} \neq 0$, which contradicts the direct decomposition $Q=E^{\prime} \oplus Q^{\prime}$.

Lemma 4.3. Let $R$ be a noetherian local ring with the maximal ideal $\mathrm{m}$. Then, for every finitely generated submodule $N$ of $E, N^{*}=0$ if and only if $N=E$.

1) This proof is suggested by $Y$. Hinohara. 
Proof. It is easily seen that a faithfully injective module is faithful (in the sense that $r Q=0$ implies $r=0$ ). Therefore $E$ is faithful and so $E^{*}=0$. Conversely let $N=\sum_{i=1}^{n} R x_{i}\left(x_{i} \in E\right)$ and $N^{*}=0$. Then we have $0=N^{*}=\bigcap_{i=1}^{n}\left(R x_{i}\right)^{*}$. Each $\left(R x_{i}\right)^{*}$ is $\mathfrak{m}$-primary ideal by [8, Lemma 3.2]. Therefore 0 is an m-primary ideal and hence $R$ must satisfy the minimum condition for ideals. Thus the annihilator relations hold between $R$ and $E$ and hence we have $N=N^{* *}=0^{*}=E$.

Lemma 4.4. Let $Q$ be any module, $N$ be its finitely generated submodule, $m$ be a maximal ideal of $R$ and $a$ be a finitely generated ideal of $R$. Then we have

(1) $\left(A n n_{Q}(a)\right)_{\mathfrak{m}}=A n n_{Q_{\mathfrak{m}}}\left(\mathfrak{a}_{\mathfrak{m}}\right)$

(2) $\left(A n n_{R}(N)\right)_{\mathfrak{m}}=A n n_{R_{\mathfrak{m}}}\left(N_{\mathfrak{m}}\right)$

Proof. We prove only (2), because (1) is proved quite similarly. Let $x=$ $[r / s]$ be any element of $\operatorname{Ann}_{R_{\mathfrak{m}}}\left(N_{\mathfrak{m}}\right)$ and $\left(n_{1}, \ldots, n_{l}\right)$ be a system of generators for $N$. Then $N_{\mathfrak{m}}$ is generated by $\left(\left[n_{1} / 1\right], \ldots,\left[n_{l} / 1\right]\right)$ over $R_{\mathfrak{m}}$. Now since $[r / s]\left[n_{i} / 1\right]=0$ for each $i$, there exist elements $s_{1}, \ldots, s_{l}$ of $R$ such that $s_{i} \notin m$ and $s_{i} r n_{i}=0$ for $i=1, \ldots, l$. Let $s^{\prime}=s_{1} s_{2} \cdots s_{l}$. Then $s^{\prime} \notin m$ and $s^{\prime} r n_{i}=0$ for each $i$, i.e. $s^{\prime} r \in \operatorname{Ann}_{R}(N)$. Thus $x=[r / s]=\left[1 / s^{\prime}\right]\left[s^{\prime} r / s\right]$ is in $\left(\operatorname{Ann}_{R}(N)\right)_{\mathfrak{m}}$, which shows $\left(\operatorname{Ann}_{R}(N)\right)_{\mathfrak{m}} \supset \operatorname{Ann}_{R_{\mathfrak{m}}}\left(N_{\mathfrak{m}}\right)$. The opposite inclusion is obvious.

THEOREM 4.5. Let $R$ be a noetherian ring. Then the annihilator relations hold between $R$ and $E$ only with respect to finitely generated submodules.

Proof. The annihilator relation for ideals follows from Theorem 4.1. Let $N$ be a finitely generated submodule of $E$. Then we have the following exact sequence $0 \rightarrow N_{\mathfrak{m}} \rightarrow\left(N^{* *}\right)_{\mathfrak{m}} \rightarrow\left(N^{* *} / N\right)_{\mathfrak{m} \rightarrow 0}$ for any maximal ideal $\mathfrak{m}$ of $R$. By Lemma 4.4 we have $\left(N^{* *}\right)_{\mathfrak{m}}=\left(N_{\mathfrak{m}}\right)^{* *}$. Therefore if we can prove $N_{\mathfrak{m}}=\left(N_{\mathfrak{m}}\right)^{* *}$, then we have $\left(N^{* * *} / N\right)_{\mathfrak{m}}=0$ for any maximal ideal $m$ and this will implies $N=N^{* *}$ by $[10, \S 9$, Lemma 2$]$. Thus, since $E_{\mathfrak{m}}=E_{R_{\mathfrak{m}}}\left(R_{\mathfrak{m}} / \mathfrak{m} R_{\mathfrak{m}}\right)^{1)}$ by [2, Cor. 1.3], we may assume that $R$ is a local ring. Let $N=R x_{1}+\cdots+R x_{n}$ where $x_{i} \in E=E(R / \mathrm{m})$. If $N^{*}=0$, then $N=E$ by Lemma 4.3 and so $N=E=E^{* *}=$ $N^{* *}$. Now we consider $N^{*} \neq 0$. Then $N$ and $N^{* *}$ are both $R / N^{*}$-modules. $R / N^{*}$ is also noetherian local and moreover we have $\operatorname{Ann}_{R / N^{*}}(N)=0$ and $N^{* *}=$ Hom $\left(R / N^{*}, E\right)=E_{R / N^{*}}\left(\left(R / N^{*}\right) /\left(\mathrm{m} / N^{*}\right)\right)$ by [1, Theorem 17]. Therefore again by Lemma 4.3 we obtain $N=E_{R / \mathrm{v}^{*}}\left(\left(R / N^{*}\right) /\left(\mathrm{m} / N^{*}\right)\right)=N^{* *}$.

1) $E_{R \mathfrak{m}}\left(\right.$ ) denotes the injective envelope considered as an $R_{\mathfrak{m}}$-module. 
COROLlary 4.6. $R$ has the minimum condition for ideals if and only if $R$ is noetherian and $E$ is finitely generated.

Proof. If $R$ is noetherian and $E$ is finitely generated, then the annihilator relations hold between $R$ and $E$ by Theorem 4.5, and so the ascending chain condition of $E$ implies the descending chain condition of $R$. The only if part is Proposition 10 of Azumaya [1].

E. Matlis [9, Proposition 3] showed that when $R$ is a complete noetherian local domain, Krull $\operatorname{dim} R=1$ if and only if every proper submodule of $E$ is finitely generated. Using Theorem 4.5 and [9, Proposition 2] (that is: $E$ has no faithful submodule if $R$ is annalytically irreducible and Krull $\operatorname{dim} R=1$ ), this result can be generalized as follows: when $R$ is a noetherian local ring, every proper submodule of $E$ is finitely generated if and only if $R$ is annalytically irreducible and Krull $\operatorname{dim} R=1$. But more generally we will show the following Theorem 4.8. To prove this theorem we need an easy

Lemma 4.7. Let $R$ be a semi-local ring. Then an R-module $A$ is finitely generated if and only if $A_{\mathfrak{m}}$ is finitely generated over $R_{\mathfrak{m}}$ for every maximal ideal $\mathrm{m}$ of $R$.

Proof. For each maximal ideal $\mathrm{m}_{i}$, we can take a system of generators $\left(\left[a_{i 1} / 1\right], \ldots,\left[a_{i n_{i}} / 1\right]\right)$ for $A_{\mathfrak{m}_{i}}$. Let $A^{\prime}$ be the submodule generated by these $a_{i j}$ 's. Then, for any $a \in A$, we can find elements $s_{i}$ of $R-m_{i}$ such that $s_{i} a \in A^{\prime}$. Since the ideal generated by these $s_{i}$ 's is equal to $R$, we obtain that $s a \in A^{\prime}$, hence $A=A^{\prime}$ is finitely generated. The converse is obvious.

THEOREM 4.8. Let $R$ be a noetherian ring such that $E(R / m)$ is not finitely generated for every maximal ideal $m$ of $R$. Then the following conditions are equivalent:

(1) Every proper submodule of $E$ not containing any $E(R / \mathrm{m})$ is finitely generated.

(2) $R$ is a semi-local ring of Krull dimension $1 . R_{\mathfrak{m}}$ is an integral domain and the annihilator relations hold between $R_{\mathfrak{m}}$ and $E_{\mathfrak{m}}=E_{R_{\mathfrak{m}}}\left(R_{\mathfrak{m}} / \mathrm{m} R_{\mathfrak{m}}\right)$ for every maximal ideal $\mathrm{m}$ of $R$.

(3) $R$ is a semi-local ring of Krull dimension $1 . R_{\mathfrak{m}}$ is an integral domain and $E_{\mathfrak{m}}$ has no proper faithful $R_{\mathfrak{m}}$-submodule for every maximal ideal $\mathfrak{m}$ of $R$.

(4) $R$ is a semi-local ring of Krull dimension 1 and $R_{\mathfrak{m}}$ is annalytically irreducible for every maximal ideal $\mathrm{m}$ of $R$. 
Proof. (1) $\Longrightarrow(2)$ : Since $\sum_{\alpha} \oplus R / \mathfrak{m}_{\alpha}$, where $\mathrm{m}_{\alpha}$ runs over all maximal ideals of $R$, is a submodule of $E$ satisfying the condition of (1), $R$ must be a semilocal ring. To see the rests of the assertion of (2) we may assume that $R$ is a local ring, since any proper $R_{\mathfrak{m}}$-submodule of $E_{\mathfrak{m}}$ is also $R_{\mathfrak{m}}$-finitely generated. The annihilator relations follow from Theorem 4.5. Let a be a non zero ideal of $R$. Then $a^{*}$ is a proper submodule of $E=E(R / \mathrm{m})$. Therefore $a^{*}$ is finitely generated. Let $\mathrm{a}^{*}=\sum_{i=1}^{n} R x_{i}\left(x_{i} \in E\right)$. Since $\left(R x_{i}\right)^{*}$ is m-primary by [8, Lemma 3.2], $\mathfrak{a}=\mathfrak{a}^{* *}=\bigcap_{i=1}^{n}\left(R x_{i}\right)^{*}$ is also m-primary. Hence we have Krull $\operatorname{dim} R=1$, if $R$ is an integral domain. If $R$ is not a domain, zero ideal is a finite intersection of non zero ideals and is also m-primary. Therefore $R$ must satisfy the minimum condition and so by Corollary $4.6 \mathrm{E}$ is finitely generated. This is a contradiction.

(2) $\Rightarrow(3)$ : Obvious.

(3) $\Longrightarrow(1)$ : Let $N$ be a proper submodule of $E$ not containing any $E(R / \mathrm{m})$. If $N_{\mathfrak{m}}=E_{\mathfrak{m}}=E(R / \mathrm{m})_{\mathfrak{m}}$, then for each $x \in E(R / \mathfrak{m})$ there exists an element $s$ of $R$ such that $s \notin \mathrm{m}$ and $s x \in N$. Since $(R x)^{*}$ is $\mathrm{m}$-primary, we have $\mathfrak{m}^{t} x=0$ for some positive integer $t$. Also we have $1=m+r s$ for some $m \in \mathrm{m}^{t}$ and $r \in$ $R$, since $R s+\mathrm{m}^{t}=R$. Therefore $x=m x+r s x=r s x \in N$ and hence we have $N \supset E(R / \mathfrak{m})$, which is a contradiction. Thus $N_{\mathfrak{m}}$ is a proper $R_{\mathfrak{m}}$-submodule of $E(R / \mathfrak{m})_{\mathfrak{m}}=E_{R_{\mathfrak{m}}}\left(R_{\mathfrak{m}} / \mathfrak{m} R_{\mathfrak{m}}\right)$. If the assertion is true for local rings, then $N_{\mathfrak{m}}$ is $R_{\mathfrak{m}}$-finitely generated and hence $N$ is $R$-finitely generated by Lemma 4.7. Thus we may assume that $R$ is a local domain of Krull dimention 1 and $E=E(R / \mathrm{m})$ has no proper faithful submodule. Now let $N$ be a proper submodule of $E$. Then $N^{*}$ is a non zero ideal and hence is m-primary. Therefore we have $N^{*}=\bigcap_{i=1}^{n} q_{i}$, where $q_{i}$ 's are irreducible m-primary ideals. By [8, Lemma 3.2] there exist $x_{i} \in E$ such that $\mathfrak{q}_{i}=\left(R x_{i}\right)^{*}$ for each $i$. Therefore $N \subset N^{* *}=$ $\left(\bigcap_{i=1}^{n} q_{i}\right)^{*}=\left(\sum_{i=1}^{n} R x_{i}\right)^{* *}=\sum_{i=1}^{n} R x_{i}$ by Theorem 4.5. Thus $N$ is finitely generated.

$(1) \Rightarrow(4)$ : We can assume that $R$ is a local ring (c.f. the proof of $(1) \Rightarrow$ (2)). Let $\hat{R}$ be the completion of $R$. Then $E$ is also the canonical injective $\hat{R}$-module by [8, Theorem 3.6]. Now, any proper $\hat{R}$-submodule of $E$ is $R$ finitely generated and hence $\hat{R}$-finitely generated. Therefore by the same way in the case of $(1) \Longrightarrow(2)$, we obtain that $\hat{R}$ is an integral domain.

$(4) \Longrightarrow(3)$ : This case is contained in [9, Proposition 2]. 


\section{REFFRENCES}

[1] G. Azumaya, A duality theory for injective modules, Amer. J. of Math., 81 (1960), pp. 249-278.

[2] H. Bass, Injective dimension in Noetherian rings. Trans. Amer. Math. Soc., 102 (1962), pp. 18-29.

[3] N. Bourbaki, Algèbre commutative, Chap. 1, (Hermann Paris, 1961).

[4] H. Cartan and S. Eilenberg, Homological algebra, (Princeton University Press, 1956).

[5] O. Goldman, Determinants in projective modules, Nagoya Math. J., 18 (1961), pp. 2736.

[6] Y. Hinohara, Projective modules over weakly Noetherian rings, J. of Math. Soc. of Japan, Vol. 15, No. 1 (1963), pp. 75-88.

[7] I. Kaplansky, Homological dimension of rings and modules, Univ. of Chicago (1959), (mimeographed notes).

[8] E. Matlis, Injective modules over Noetherian rings, Pacific J. of Math., 8 (1958), pp. 511-528.

[9] E. Matlis, Some properties of Noetherian domain of dimension one, Canadian J. of Math. 13 (1961), pp. 569-586.

[10] D. G. Northcott, An introduction to homological algebra, (Cambridge University Press, 1960),

Tokyo Metropolitan University 